\title{
Fiber-optic acoustic pressure sensor based on large-area nanolayer silver diaghragm
}

\author{
Feng Xu, ${ }^{1}$ Jinhui Shi, ${ }^{1}$ Kui Gong, ${ }^{1}$ Hefei Li, ${ }^{1}$ Rongqing Hui, ${ }^{2}$ and Benli $\mathbf{Y u}^{1, *}$ \\ ${ }^{1}$ School of Physics and Materials Science, Anhui University, Hefei 230039, China \\ ${ }^{2}$ Department of Electrical Engineering and Computer Science, University of Kansas, Lawrence, Kansas 66045, USA \\ *Corresponding author: benliyu@ahu.edu.cn
}

Received February 6, 2014; revised April 2, 2014; accepted April 2, 2014;

posted April 9, 2014 (Doc. ID 206057); published May 5, 2014

\begin{abstract}
A fiber-optic acoustic pressure sensor based on a large-area nanolayer silver diaphragm is demonstrated with a high dynamic pressure sensitivity of $160 \mathrm{~nm} / \mathrm{Pa}$ at $4 \mathrm{kHz}$ frequency. The sensor exhibits a noise limited detectable pressure level of $14.5 \mu \mathrm{Pa} / \mathrm{Hz}^{1 / 2}$. Its high dynamic pressure sensitivity and simple fabrication process make it an attractive tool for acoustic sensing and photo-acoustic spectroscopy. (c) 2014 Optical Society of America

OCIS codes: (060.2370) Fiber optics sensors; (120.2230) Fabry-Perot.

http://dx.doi.org/10.1364/OL.39.002838
\end{abstract}

In recent years, fiber-optic pressure or acoustic sensors based on sensing diaphragms have attracted much interest because of their unique advantages over traditional sensors. These advantages include immunity to electromagnetic interference, the capability of performing remote sensing, very high resolution, fast response, and compact size [1]. In general, such a pressure sensor based on a micro Fabry-Perot (FP) cavity commonly uses an elastic diaphragm attached at the tip of an optical fiber, and the diaphragm as one of the mirrors of the FP cavity can be made of various materials, such as silica [2], polymer [3] , silver, and graphene [4, $\underline{5}$ ]. The FP pressure sensors based on different diaphragms have been investigated intensively in recent years, and the sensitivity has been improved steadily. Recently, Ma et al. demonstrated a Fabry-Perot interferometer (FPI) acoustic sensor based on a multilayer graphene diaphragm with thickness of approximately $100 \mathrm{~nm}$, and dynamic pressure sensitivity of $1100 \mathrm{~nm} / \mathrm{kPa}$ was achieved with the diaphragm diameter of $125 \mu \mathrm{m}$ [6]. Although the pressure sensitivity of the sensor based on the graphene diaphragm can potentially be very high thanks to small thickness, it is usually difficult to prepare and process single- or few-layered graphene diaphragms with large diameters and transfer them onto the sensor head. In addition, optical reflectivity of the graphene layer is relatively small compared to metal films.

In this Letter, we demonstrate an acoustic sensor based on a large area silver diaphragm $150 \mathrm{~nm}$ in thickness and $2.4 \mathrm{~mm}$ in diameter. While the thickness is comparable to the graphite-based diaphragms previously reported [ $\underline{6}$ ], the diameter is an order of magnitude larger. The acoustic pressure test shows that such a sensor based on a large area silver diaphragm has a sensitivity of up to $160 \mathrm{~nm} / \mathrm{Pa}$ at $4 \mathrm{kHz}$ frequency. To our best knowledge, this is the highest acoustic pressure sensitivity ever reported for fiber-optic acoustic sensors based on diaphragms. The sensor is able to detect acoustic pressure as weak as $14.5 \mu \mathrm{Pa} / \mathrm{Hz}^{1 / 2}$.

The acoustic pressure sensing head based on the silver diaphragm comprises a standard polarization maintaining fiber (PM fiber), a ceramic split sleeve, and the silver diaphragm, as shown in Fig. 1(a). Figure 1(b) is the image of the proposed sensing head. A picture of the ceramic split sleeve, on which the diaphragm is attached, is also shown in the bottom inset of Fig. 1(b), and the inner diameter of this split sleeve is $2.4 \mathrm{~mm}$. The PM fiber end facet was polished with an angle of $8^{\circ}$ to reduce the Fresnel reflection from the fiber end surface. The fabrication process of the sensing head is similar to that reported in our previous paper [5]. The distance between the fiber end and the diaphragm is approximately $150 \mu \mathrm{m}$ adjusted by a high precision linear stage (Newport ILS-250CC) with a displacement resolution of $0.5 \mu \mathrm{m}$.

The silver thin film used in this sensor is approximately $150 \mathrm{~nm}$ thick with $>2.4 \mathrm{~mm}$ diameter that covers one end of the split sleeve. The top inset of Fig. 1(b) is an SEM image of the silver diaphragm. This sensing diaphragm has several advantages, including good stability and high reflectivity over the near infrared wavelength range. In general, the pressure sensitivity depends both on the material and the thickness of the diaphragm. In fact, the pressure sensitivity of an acoustic sensor based on an edge-clamped circular diaphragm is proportional to $R^{4} / t^{3}$, where $R$ is the radius and $t$ is the thickness of the diaphragm [7]. Therefore a diaphragm with large
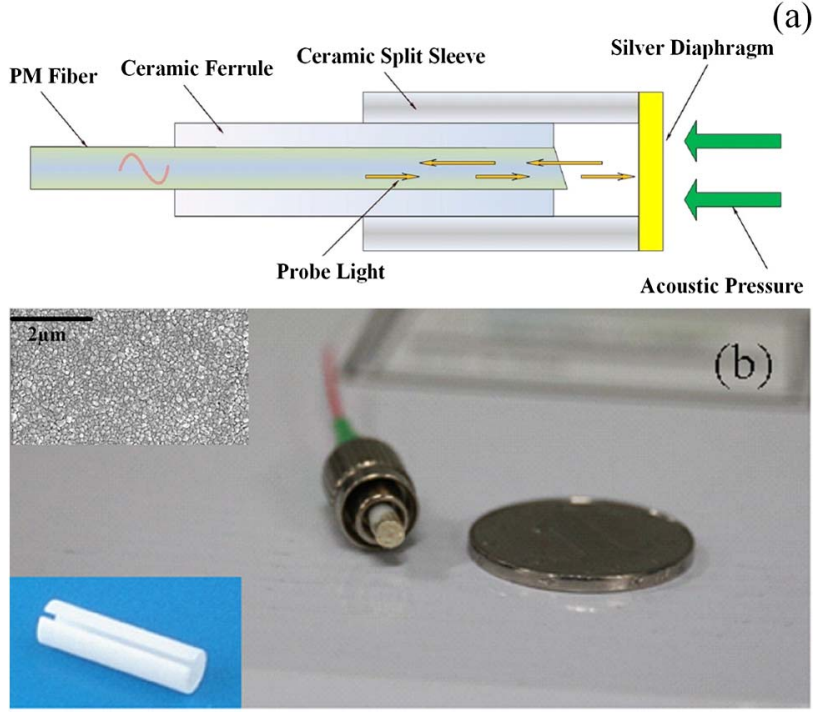

Fig. 1. (a) Schematic and (b) image of the sensing head. 
radius and small thickness will help improve the sensitivity.

In our experiment, the electroless plating method was used to fabricate the silver nanofilm as the sensing diaphragm. This fabrication process is relatively simple and low cost compared with the microelectromechnical technique, often used in the fabrication of thin silicon-based sensing diaphragms [2]. It is rather straightforward to obtain the silver film thickness down to nanometers compared with other fabrication processes based on polymer and silica materials. The details of the electroless plating fabrication process can be found in our prior publication [5]. The typical thickness of the prepared silver diaphragm is $150 \mathrm{~nm}$ with excellent uniformity, and the reflectivity of the silver diaphragm is higher than $90 \%$ over the wavelength range from 1.5 to $1.6 \mu \mathrm{m}$.

To test the dynamic pressure sensitivity of such a silver nanolayer diaphragm, a vibration exciter (B\&K 4809) and a piezoelectric accelerometer (B\&K 8305) were used to calibrate the relationship between the demodulated output voltage signal and the applied vibration amplitude. In the calibration process, the vibration exciter was driven by a sinusoid waveform and its vibration amplitude was directly measured. In the experimental setup schematically shown in Fig. 2, a laser source with $1541.1 \mathrm{~nm}$ wavelength is launched into a 3-dB (50:50) fiber coupler, where the input optical signal is divided into two arms. One of them is the reference arm, in which the reference light is phase modulated by a piezoelectric transducer at $41.2 \mathrm{kHz}$. The other arm is the sensing arm, in which the sensing light is redirected by a fiber-optic circulator and reflected by a mirror attached on the vibration exciter. The reflected optical signal and the reference light recombine at the output coupler and detected by an optical receiver where the vibration signature can be demodulated by the phase generated carrier (PGC) method [8]. In order to reduce the polarization induced fading, all fiber-optic devices are polarization maintaining. Figure 3 shows the demodulated output voltage signal that linearly increases with the vibration amplitude of the exciter with a slop of $8.44 \mathrm{mV} / \mathrm{nm}$. It also has a good linearity with a correlation coefficient of 0.9982 . This slop is then used as the system calibration base for the sensitivity measurement of the sensor head.

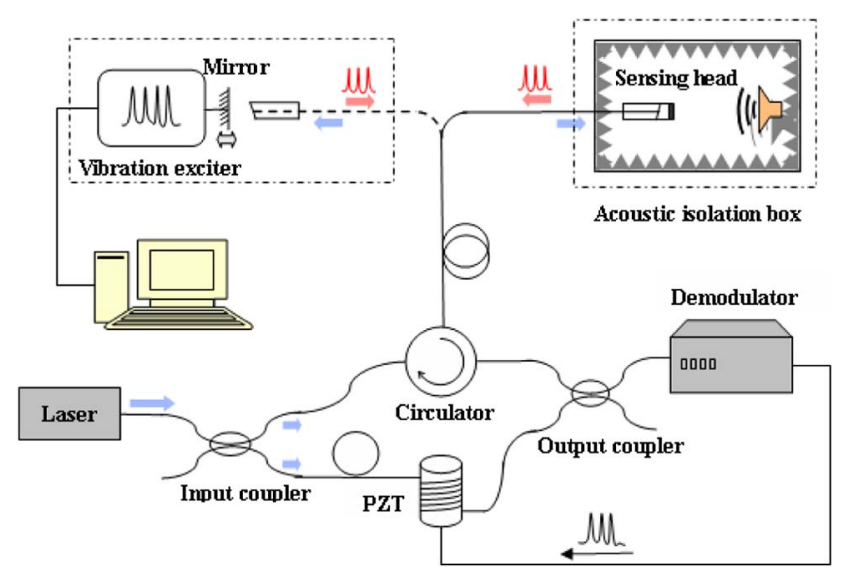

Fig. 2. Experimental setup of the calibration and acoustic pressure test.

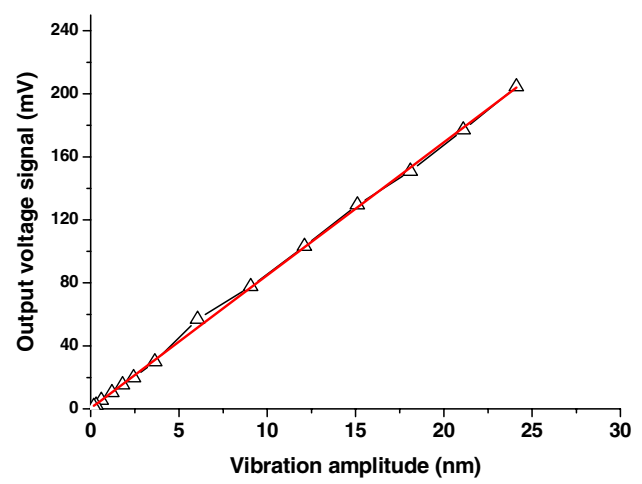

Fig. 3. Dependence of output voltage signal on the vibration amplitude.

We then add the sensing head into the system that is place into an acoustic isolation box (B\&K 4232), in which a calibrated microphone (B\&K 4192) is used to calibrate the acoustic pressure signal as shown on the top right of Fig. 2. This setup allows the testing of dynamic pressure response of the silver diaphragm with varying acoustic pressure levels applied through a speaker. The amplitude of the induced vibration on the silver diaphragm corresponding to each acoustic pressure level was measured as shown in Fig. 4, where the frequency of the acoustic pressure signal was $4 \mathrm{kHz}$. The slope of this curve indicates that the dynamic response of the silver diaphragm has a sensitivity of $160 \mathrm{~nm} / \mathrm{Pa}$ at $4 \mathrm{kHz}$. This sensitivity is approximately two orders of magnitude higher than that reported using graphite diaphragm [6]. This sensitivity improvement is primarily attributed to the significantly increased diameter of the diaphragm. To the best of our knowledge, this is the highest sensitivity of dynamic pressure response ever reported with fiber-optic acoustic sensors based on sensing diaphragms.

Figure 5 shows the electrical power spectrum measured by $\mathrm{a} B \mathrm{~B}$ acoustic analyzer, which was obtained when $3 \mathrm{mPa}$ acoustic pressure at $4 \mathrm{kHz}$ was applied on the sensing head. The output signal shows a signalto-noise ratio (SNR) of $43.3 \mathrm{~dB}$ with $2 \mathrm{~Hz}$ spectral resolution. The noise floor is about $-91.5 \mathrm{dBm}$. Therefore, the noise-limited minimum detectable pressure level in this system is about $14.5 \mu \mathrm{Pa} / \mathrm{Hz}^{1 / 2}$. In our system, the received optical signal was approximately $-20 \mathrm{dBm}$, which can be increased by using a laser source with higher power if SNR becomes a limiting factor.

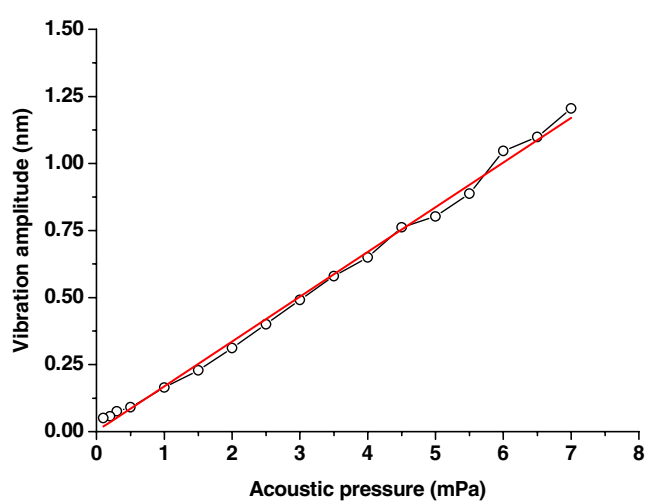

Fig. 4. Vibration amplitude of the silver diaphragm for varying applied acoustic pressure level at $4 \mathrm{kHz}$. 


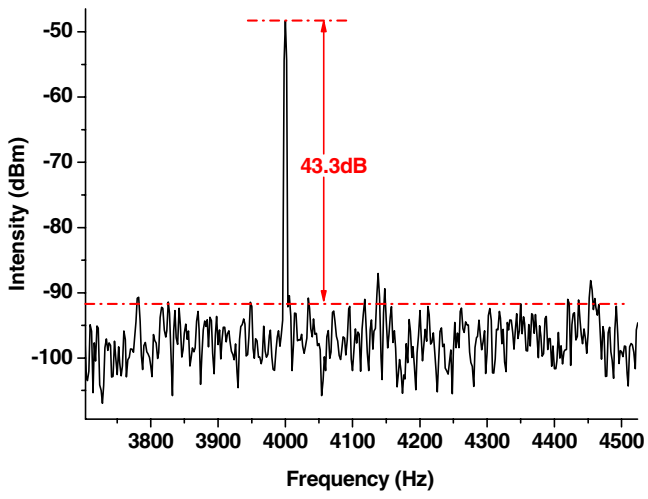

Fig. 5. Power spectrum of the sensing head when $3 \mathrm{mPa}$ acoustic pressure was applied at $4 \mathrm{kHz}$ frequency.

In addition to dynamic pressure sensitivity, frequency response is another important parameter for acoustic sensors. Theoretically, for a clamped circular diaphragm, the resonance frequency, $f$, is inversely proportional to the square of the radius $R$ of the diaphragm, and therefore the resonance frequency will decrease with the increasing of $R$. Figure 6 shows the measured frequency response at an acoustic pressure of $2 \mathrm{mPa}$, which exhibits a dominant resonance peak at approximately $800 \mathrm{~Hz}$ and several high order resonance peaks. Although the strong frequency dependence of the response is unfavorable for the application as a microphone, the significantly improved pressure sensitivity makes this sensor ideal for photo-acoustic signal sensing in which the acoustic frequency is predetermined. Note that the dynamic pressure sensitivity at the peak resonance frequency is approximately $2100 \mathrm{~nm} / \mathrm{Pa}$, more than an order of magnitude higher than that measured at $4 \mathrm{kHz}$, which is advantageous for frequency selective sensing.

In conclusion, we have demonstrated a fiber-optic acoustic sensor based on a large area nanolayer silver diaphragm. The record high dynamic pressure sensitivity of $160 \mathrm{~nm} / \mathrm{Pa}$ at $4 \mathrm{kHz}$ is primarily attributed to the large diameter and small thickness of the diaphragm. This sensitivity can be much higher at the peak resonance frequency, which is useful for frequency-selective photoacoustic signal sensing. Because of its low cost and

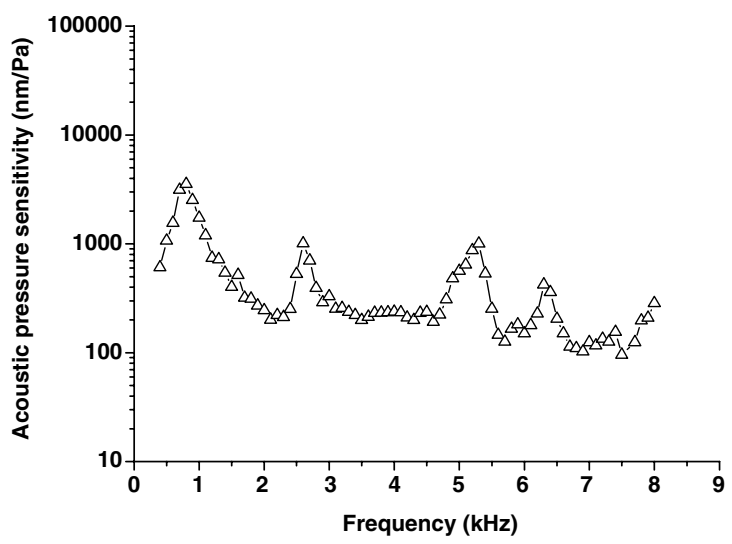

Fig. 6. Dependence of the output signal voltage on frequency when $2 \mathrm{mPa}$ acoustic pressure was applied on the sensing head.

simple fabrication process, fiber-optic acoustic pressure sensors based on silver nanofilm have great potential in acoustic sensing and photo-acoustic spectroscopy.

This work was supported by the National Natural Science Foundation of China under grant 61108076, Anhui Provincial Natural Science Foundation under grant 1308085MF91, the Key Research Project of Anhui Education Department under grant KJ2011A009, and by the 211 Project of Anhui University.

\section{References}

1. Y. Rao, Opt. Fiber Technol. 12, 227 (2006).

2. W. Wang, N. Wu, Y. Tian, C. Niezrecki, and X. Wang, Opt. Express 18, 9006 (2010).

3. L. H. Chen, C. C. Chan, W. Yuan, S. K. Goh, and J. Sun, Sens. Actuators A 163, 42 (2010).

4. J. Ma, W. Jin, H. L. Ho, and J. Y. Dai, Opt. Lett. 37, 2493 (2012).

5. F. Xu, D. Ren, X. Shi, C. Li, W. Lu, L. Lu, L. Lu, and B. Yu, Opt. Lett. 37, 133 (2012).

6. J. Ma, H. Xuan, H. L. Ho, W. Jin, Y. Yang, and S. Fan, IEEE Photon. Technol. Lett. 25, 932 (2013).

7. B. Yu, D. W. Kim, J. Deng, H. Xiao, and A. Wang, Appl. Opt. 42, 3241 (2003).

8. A. Dandridge, A. B. Tveten, and T. G. Giallorenzi, IEEE Trans. Microwave Theory Tech. 30, 1635 (1982). 